\title{
Chronic intermittent hypoxia mimicking sleep apnoea increases spontaneous tumorigenesis in mice
}

\author{
To the Editor:
}

Obstructive sleep apnoea (OSA) is a very prevalent disorder with well proven mid- and long-term deleterious consequences, such as increased risk of cardiovascular, metabolic and neurocognitive diseases [1]. Recently, considerable data from both animal models and patient studies also suggest that OSA increases the risk of cancer incidence and mortality [2]. Some of these studies indicate that nocturnal hypoxic events experienced by OSA patients as a consequence of recurrent upper airway obstructions may be a main challenge driving tumour progression [3,4]. Although the clinical and experimental data available do not undoubtedly prove a relationship between cancer and OSA [5], its plausibility has raised the interest of basic and clinical researchers in the field, warranting further investigation [6].

The experimental data available in murine models consistently indicate that intermittent hypoxia at a rate and magnitude simulating the pattern experienced by patients with severe OSA increases tumour growth rate, invasion and metastasis, specifically in melanoma and lung cancer cell models [7-9]. These data also suggest that several pathophysiological processes are involved in boosting cancer enhancement by intermittent hypoxia. For instance, alterations in the immune response by increases in the pro-tumoral phenotype of tumour associated macrophages, enhanced recruitment and polarisation of adipose tissue macrophages, alteration of the adrenergic pathway, increase of circulating tumour DNA in peripheral blood and increased release of exosomes [2, 4, 10, 11].

Nevertheless, all available animal model data relating to OSA and cancer have been obtained by artificially implanting cancer cells into a healthy animal and investigating how and why tumour progresses under intermittent hypoxia as compared with normoxia. This experimental information gives important clues to interpret the increase in cancer progression observed in patients with OSA, but does not inform on whether intermittent hypoxia per se increases the transformation of normal cells into tumour cells, i.e. tumorigenesis, a process that could be theoretically expected from the oxidative stress suffered by tissues in OSA patients as a consequence of hypoxia/reoxygenation [12]. New evidence on intermittent hypoxia-induced tumorigenesis would be most useful to interpret clinical data concerning incidence of new cancers in OSA patients. Accordingly, here we report the results from a study showing, for the first time, that intermittent hypoxia mimicking severe OSA does increase the appearance of spontaneous tumours observed in otherwise not manipulated animals.

The study, approved by the ethical committee of the University of Valladolid (Valladolid, Spain), was performed on male outbred Swiss CD1 mice obtained from Janvier (Le Genest-Saint-Isle, France), aged 3 months and maintained to the age of 15 months in the conventional animal facilities of the university, subjected to a regular regime (standard cages, $20-24^{\circ} \mathrm{C}, 12 \mathrm{~h} \mathrm{light/day,} \mathrm{water} \mathrm{and} \mathrm{food} \mathrm{ad} \mathrm{libitum).} \mathrm{Mice}$ (aged 15 months; $\mathrm{n}=200$ ) were randomly divided into three groups: a normoxic control group breathing room air $(n=71)$ and two groups subjected to chronic intermittent hypoxia at a rate corresponding to 30 events $\cdot \mathrm{h}^{-1}$ (breathing cycles of $40 \mathrm{~s}$ of hypoxic air and $80 \mathrm{~s}$ of room air), $8 \mathrm{~h} \cdot$ day $^{-1}(08: 00-16: 00 \mathrm{~h})$ over 3 months to the age of 18 months. The intermittent hypoxia exposures were mild $\left(12 \%\right.$ oxygen $\left(\mathrm{O}_{2}\right)$ nadir; $\mathrm{n}=61)$ and more severe $\left(7.5 \% \mathrm{O}_{2}\right.$ nadir; $\left.\mathrm{n}=68\right)$ (figure $\left.1 \mathrm{a}\right)$. These patterns of breathing caused nadir mean \pm SE values in arterial oxygen saturation measured by pulse oximetry $\left(\mathrm{SpO}_{2}\right)$ of $74.3 \pm 2.5 \%$ and $55.5 \pm 4.8 \%$, respectively, as in two additional groups each including six anaesthetised mice (figure $1 \mathrm{~b}$ ). However, owing

@ERSpublications

Chronic intermittent hypoxia mimicking sleep apnoea increases spontaneous tumorigenesis in mice http://ow.ly/BoF6306AnI0

Cite this article as: Gallego-Martin T, Farré R, Almendros I, et al. Chronic intermittent hypoxia mimicking sleep apnoea increases spontaneous tumorigenesis in mice. Eur Respir J 2017; 49: 1602111 [https://doi.org/10.1183/13993003.02111-2016]. 

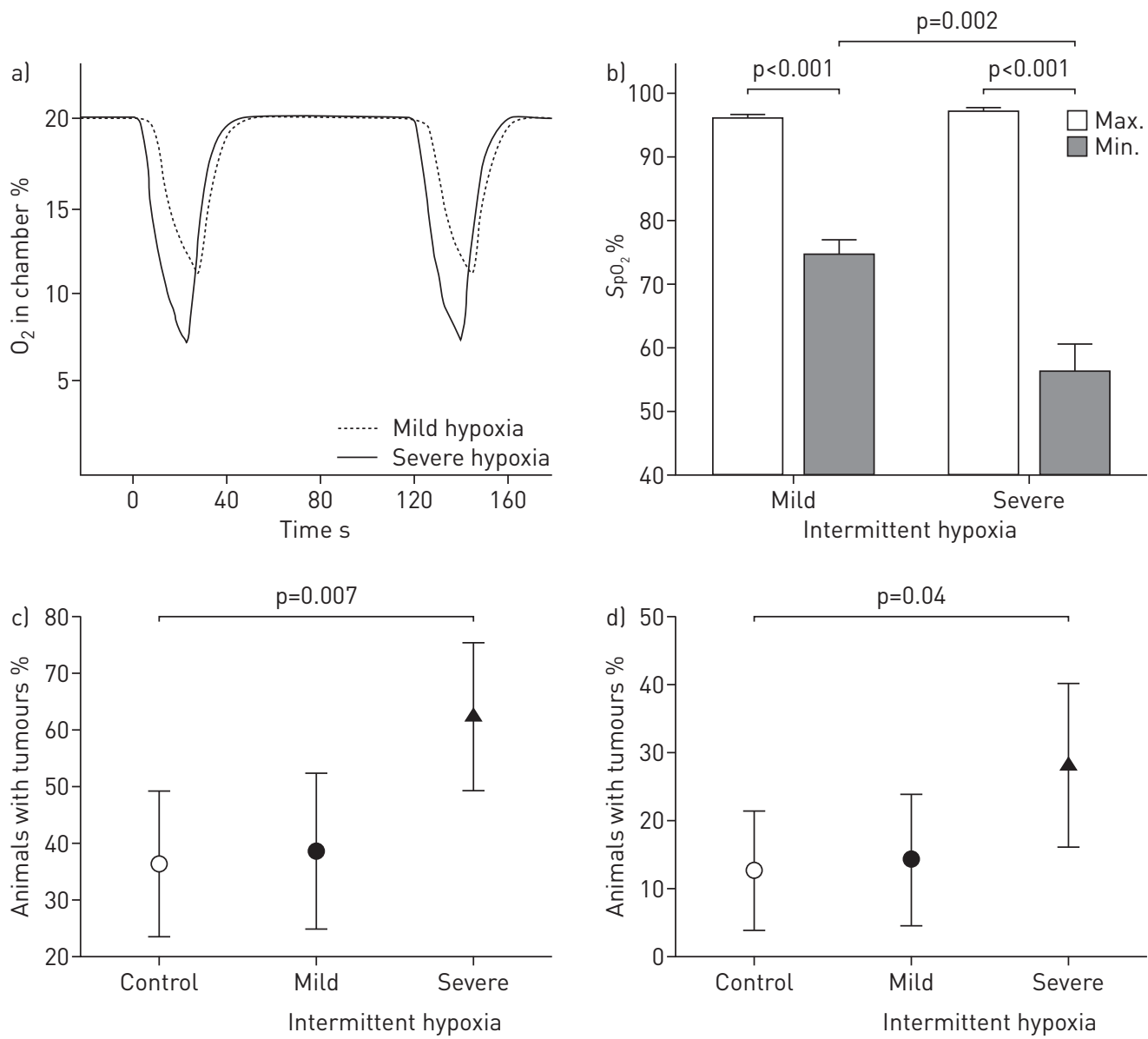

FIGURE 1 a) Pattern of oxygen $\left(\mathrm{O}_{2}\right)$ concentration in the air breathed by mice subjected to mild and severe intermittent hypoxia. b) Nadir (minimum) and recovery (maximum) in the cycles of arterial oxygen saturation $\left(\mathrm{SpO}_{2}\right)$ caused by breathing the mild and severe intermittent hypoxia patterns. Data are presented as mean $\pm \mathrm{SE}$. Incidence $(95 \% \mathrm{Cl}$ ) of c) any type of tumour and d) lung tumours in aged mice after 3 months' exposure to normoxia (control), mild and severe intermittent hypoxia.

to potential slight respiratory depression during anaesthesia, it could be expected that desaturations in awake animals are less severe than in figure $1 \mathrm{~b}$. At the end of intermittent hypoxia or normoxia exposure the animals were euthanised and subjected to necropsy. For qualitative variables, percentages (and 95\% confidence intervals) within specified groups were calculated and p-values comparing controls and intermittent hypoxia were derived using Chi-squared tests. For quantitative variables, one-way ANOVA was employed to assess differences between controls and intermittent hypoxia groups. $\mathrm{p}<0.05$ was considered the statistical significance threshold.

The survival rate at 18 months was consistent with that expected in this mouse strain [13] and was not different among the three groups: $77.5 \pm 9.7 \%$ (control), $80.3 \pm 10.0 \%$ (mild intermittent hypoxia) and $77.9 \pm 9.9 \%$ (severe intermittent hypoxia). Necropsies found tumours in skin, liver and lung. The percentage of control animals exhibiting tumours in at least one organ was $36.4 \pm 12.7 \%$, a figure in keeping with data for this age and mouse strain [13]. For mice under mild and severe intermittent hypoxia this percentage was $38.8 \pm 13.6 \%$ and $62.3 \pm 13.0 \%$, respectively, and the difference between controls and severe intermittent hypoxia was statistically significant (figure 1c). There were no significant differences in the appearance of liver or skin tumours. The lack of increased skin tumorigenesis under intermittent hypoxia contrasts with previously reported augmentation in the growth of implanted melanoma tumours $[7,8]$, suggesting that the effects of this OSA-mimicking challenge on de novo malignancy induction and on tumour aggressiveness could be different. Remarkably, lung tumours showed significant higher prevalence in the group subjected to severe intermittent hypoxia compared with controls (figure 1d). All lung tumours were peripheral masses which involved subsegmental bronchi. Histologically, they presented as organoid nesting, trabecular growth and perilobular palisading patterns, consistent with neuroendocrine tumours. No significant differences were found in the size of lung tumours between groups: $3.08 \pm 1.28 \mathrm{~mm}$, $2.71 \pm 1.03 \mathrm{~mm}$ and $3.79 \pm 4.26 \mathrm{~mm}$ for controls, mild and severe intermittent hypoxia, respectively. Liver 
tumours were of polygonal cells with a predominantly trabecular pattern consistent with hepatocellular carcinoma. Skin lesions ranged from ulceration with granulation tissue to lesions with necrosis, increased vascularisation and atypical fusiform cell proliferations. The fact that the increase in tumorigenesis induced by intermittent hypoxia affected several organs differently could reflect a variation in cell/tissue type response to this challenge.

This study is unique, since it shows for the first time that relatively severe, but not mild, intermittent hypoxia increases spontaneous tumorigenesis. Moreover, some specific features of the model are noticeable. First, the rate of intermittent hypoxia employed, 30 events $\cdot \mathrm{h}^{-1}$, was considerable but in no way exceptional from the clinical perspective. In addition, the nadir of hypoxic events resulted in $\mathrm{SpO}_{2}$ minima that are comparable to the ones measured in mild and severe OSA patients. In fact, both the rate and magnitude of hypoxia in this study were less strenuous than in several previous studies using artificially induced tumours in OSA models (e.g. $5 \% \mathrm{O}_{2}$ nadir and 60 events.h ${ }^{-1}$ ) [6, 7]. Second, contrary to most previous animal studies on OSA and cancer in which young animal mice were investigated [6-11], here we used mice at an age which mimics human maturity. Indeed, the age of the mice in this study (15-18 months) is equivalent to human 50-60 years [14], an age with clinical interest regarding the prevalence of OSA and cancer. Third, this study was performed in an outbred mouse strain (CD-1) presenting polymorphisms at a considerable number of loci and displaying patterns of linkage disequilibrium and heterogeneity similar to wild-caught mice [15], being therefore particularly interesting animals to model actual human genetic heterogeneity.

In conclusion, we here provide novel evidence suggesting that OSA, via intermittent hypoxia, could increase the spontaneous tumorigenesis associated with normal ageing. In addition to providing additional support for interpreting clinical data on cancer incidence in OSA, this study paves the way to future research on the mechanisms involved in the process and also to investigate how intermittent hypoxia modulates the well-known tumorigenesis induced by chemical and physical carcinogenic agents challenging human health, particularly as a result of ageing.

Teresa Gallego-Martin ${ }^{1,2}$, Ramón Farré ${ }^{2,3}$, Isaac Almendros ${ }^{2,3}$, Elvira Gonzalez-Obeso ${ }^{4}$ and Ana Obeso ${ }^{1,2}$ ${ }^{1}$ Departamento de Bioquímica y Biología Molecular y Fisiología, Facultad de Medicina, Universidad de Valladolid, IBGM/CSIC, Valladolid, Spain. ${ }^{2}$ CIBERES, Instituto de Salud Carlos III, Madrid, Spain. ${ }^{3}$ Unitat de Biofísica i Bioenginyeria, Facultat de Medicina, Universitat de Barcelona-IDIBAPS, Barcelona, Spain. ${ }^{4}$ Servicio de Anatomía Patológica, Hospital Clínico Universitario, Valladolid, Spain.

Correspondence: Ana Obeso, Departamento de Bioquímica y Biología Molecular y Fisiología, Facultad de Medicina, Ramón y Cajal, 7, 47005 Valladolid, Spain. E-mail: aobeso@ibgm.uva.es

Received: Oct 282016 | Accepted after revision: Nov 192016

Support statement: CIBER de Enfermedades Respiratorias (CB06/06/0050); Spanish Ministry of Economy and Competitiveness, Instituto de Salud Carlos III, MINECO/FEDER, UE (BFU2015-70616-R, PI2014-00004); and Spanish Association Against Cancer (APRO-I Valladolid). Funding information for this article has been deposited with the Open Funder Registry.

Conflict of interest: None declared.

Acknowledgements: The authors thank Ana Gordillo (Universidad de Valladolid/CIBERES, Valladolid, Spain) for her technical support.

\section{References}

1 Mokhlesi B, Ham SA, Gozal D. The effect of sex and age on the comorbidity burden of OSA: an observational analysis from a large nationwide US health claims database. Eur Respir J 2016; 47: 1162-1169.

2 Gozal D, Farré R, Nieto FJ. Obstructive sleep apnea and cancer: epidemiologic links and theoretical biological constructs. Sleep Med Rev 2016; 27: 43-55.

3 Martínez-García MA, Martorell-Calatayud A, Nagore E, et al. Association between sleep disordered breathing and aggressiveness markers of malignant cutaneous melanoma. Eur Respir J 2014; 43: 1661-1668.

4 Gozal D, Farré R, Nieto FJ. Putative links between sleep apnea and cancer: from hypotheses to evolving evidence. Chest 2015; 148: 1140-1147.

5 Gozal D, Ham SA, Mokhlesi B. Sleep apnea and cancer: analysis of a nationwide population sample. Sleep 2016; 39: 1493-1500.

6 Lévy P, Godin-Ribuot D, Pepin JL. Sleep apnoea and cancer: the new challenge. Eur Respir J 2014; 43: 1567-1570.

7 Almendros I, Montserrat JM, Ramírez J, et al. Intermittent hypoxia enhances cancer progression in a mouse model of sleep apnoea. Eur Respir J 2012; 39: 215-217.

8 Almendros I, Montserrat JM, Torres M, et al. Intermittent hypoxia increases melanoma metastasis to the lung in a mouse model of sleep apnea. Respir Physiol Neurobiol 2013; 186: 303-307.

9 Almendros I, Wang Y, Becker L, et al. Intermittent hypoxia-induced changes in tumor-associated macrophages and tumor malignancy in a mouse model of sleep apnea. Am J Respir Crit Care Med 2014; 189: 593-601.

10 Almendros I, Gileles-Hillel A, Khalyfa A, et al. Adipose tissue macrophage polarization by intermittent hypoxia in a mouse model of OSA: effect of tumor microenvironment. Cancer Lett 2015; 361: 233-239. 
11 Almendros I, Khalyfa A, Trzepizur W, et al. Tumor cell malignant properties are enhanced by circulating exosomes in sleep apnea. Chest 2016; 150: 1030-1041.

12 Quintero M, Gonzalez-Martin MC, Vega-Agapito V, et al. The effects of intermittent hypoxia on redox status, NF- $\mathrm{KB}$ activation, and plasma lipid levels are dependent on the lowest oxygen saturation. Free Radic Biol Med 2013; 65: 1142-1154.

13 Giknis MLA, Clifford CB. Spontaneous Neoplastic Lesions in the Crl:CD1 (ICR) Mouse in Control Groups from 18 Month to 2 Year Studies. Charles River Laboratories. www.criver.com/files/pdfs/rms/cd1/rm_rm_r_cdl_ mouse_tox_data_2010.aspx Date last accessed: October 8, 2016. Date last updated: March 2010.

14 Flurkey K, Currer M, Harrison DE. Mouse models in aging research. In: Fox JG, Davisson MT, Quimby FW, et al., eds. The Mouse in Biomedical Research. 2nd Edn. Burlington, Academic Press, 2007; pp. 637-672.

15 Aldinger KA, Sokoloff G, Rosenberg DM, et al. Genetic variation and population substructure in outbred CD-1 mice: implications for genome-wide association studies. PLoS One 2009; 4: e4729.

Copyright @ERS 2017 